\title{
Preparation and Evaluation of Inclusion Complex of the Lipid Lowering Drug Lovastatin with $\beta$-Cyclodextrin
}

\author{
R. P. Patel and M. M. Patel \\ Department of Pharmaceutics, S.K. Patel College of Pharmaceutical Education and Research, Ganpat \\ University, Ganpat vidyanagar, Kherva, Mehsana-Gozaria Highway, PIN-390 001, Gujarat, India.
}

\begin{abstract}
Several attempts have been made to improve the solubility of water insoluble drugs. Over the years, inclusion complexation of drugs with $\beta$-cyclodextrin has emerged as a viable attempt to improve the dissolution of water insoluble drugs. The aim of the present work was to improve the dissolution rate of lovastatin, a water insoluble drug, by inclusion complexation with $\beta$-cyclodextrin. The stoichiometric ratio determined by phase solubility analysis for inclusion complexation of lovastatin with $\beta$-cyclodextrin was $1: 1$. The solubility of lovastatin increased with increasing amount of $\beta$-cyclodextrin in water. Gibbs free energy $\left(\Delta \mathrm{G}_{\mathrm{tr}}^{\circ}\right)$ values were all negative, indicating the spontaneous nature of lovastatin solubilization. Complexes of lovastatin were prepared with $\beta$-cyclodextrin by various methods such as kneading, coevaporation and physical mixing. The complexes were characterized by Fourier-transform infrared (FTIR) spectroscopy, differential scanning calorimetry (DSC), and X-ray diffraction (XRD) patterns. These studies indicated the inclusion of lovastatin in the cavity of $\beta$-cyclodextrin. The complexation resulted in a marked improvement in the solubility of lovastatin. The complex prepared by kneading method showed fastest and highest in vitro dissolution rate compared to the tablets of pure of lovastatin. Physical mixture of $\beta$ cyclodextrin/lovastatin also showed significant improvement in the dissolution rate compared to pure lovastatin. Mean dissolution time (MDT) of lovastatin decreased significantly after preparation of complexes and physical mixture of lovastatin with $\beta$-cyclodextrin. Similarity factor $\left(f_{2}\right)$ indicated significant difference between the release profiles of lovastatin from complexes and from pure lovastatin.
\end{abstract}

Key words: Lovastatin, $\beta$-cyclodextrin, inclusion complexation, in vitro dissolution studies.

\section{INTRODUCTION}

Cyclodextrins (CDs) form a group of structurally related oligosaccharides with cylinder-shaped cavities that have the capacity to form inclusion complexes with many drugs by taking a whole drug molecule, or a part of it, into the cavity. ${ }^{1,2}$ Because of the large number of hydroxyl groups on CDs, they are water-soluble. They are known for their ability to molecularly encapsulate a wide variety of drugs into their hydrophobic cavity without the formation of any covalent bonds. ${ }^{3-6}$ Complexation with cyclodextrins

Correspondence to: Rakesh P. Patel

E-mail: raka_77us@yahoo.com

Dhaka Univ. J. Pharm. Sci. 6(1): 25-36, 2007 (June) has been reported to enhance the solubility, dissolution rate and bioavailability of poorly water soluble drugs. CDs first came to the fore in marketed products as drug delivery technologies that enabled the development of various prostaglandins. ${ }^{7}$ Many other drugs have been tested for $\mathrm{CD}$ inclusion to enhance solubility such as bropirimine, ibuprofen, tolbutamide doxorubicin and daunorubicin. ${ }^{8-11}$

In vitro dissolution testing provides an easy and convenient means to evaluate the performance of pharmaceutical preparations. In this study an attempt was made to compare the similarity between in vitro dissolution profiles of Lovastatin (Lov) from complexes, physical mixture and pure Lov. 
Dissolution profiles can be compared by calculating similarity factor $\left(f_{2}\right.$ values). The method was first reported by Moore and Flanner. ${ }^{12}$

Mean dissolution time (MDT) reflects the time for the drug to dissolve and is the first statistical moment for the cumulative dissolution process that provides an accurate drug release rate. ${ }^{13}$

Lov is a prodrug. After oral administration, the inactive parent lactone is hydrolyzed to the corresponding hydroxyacid form. The hydroxyacid is the principal metabolite and a potent inhibitor of 3hydroxy-3-methylglutaryl-coenzyme A (HMG CoA) reductase. This enzyme catalyzes the conversion of hydroxymethylglutarate to mevalonate, which is early and rate limiting step in the biosynthesis of cholesterol. $^{14,15}$ Lov is white crystalline powder, insoluble in water $(0.4 \mu \mathrm{g} / \mathrm{ml})$. At room temperature, the partition coefficient of Lov in n-octanol/water system is approximately $\mathrm{K}_{\mathrm{o} / \mathrm{w}}=1.2 \times 10^{4} .{ }^{16}$ Low aqueous solubility of Lov leads to inadequate dissolution in gastrointestinal (GI) fluids and hence poor absorption, distribution and target organ delivery. Improvement of aqueous solubility in such a case is a valuable goal to improve therapeutic efficacy. The present study was planned to improve the aqueous solubility and dissolution rate of Lov by preparing complexes with $\beta$-cyclodextrin $(\beta-\mathrm{CD})$ employing methods such as kneading and coevaporation. The study further aimed to characterize the interaction between Lov and $\beta-C D$.

\section{MATERIALS AND METHODS}

Materials. Lov was received as a gift sample from Lincoln Pharmaceuticals Ltd, (Ahmedabad, India). The samples of $\beta-\mathrm{CD}$ and sodium lauryl sulfate (SLS) were procured from S.D. Fine Chemicals, (Vadodadra, India). Directly compressible lactose, maize starch, sodium starch glycollate, colloidal silicon dioxide, and magnesium stearate were received as gift samples from Maan Pharmaceuticals Ltd., (Ahmedabad, India). All chemicals and solvents used in this study were of analytical reagent grade. Freshly distilled water was used throughout the work.

Phase Solubility Study. The stoichiometric ratio and constant were determined by using phase solubility method of Higuchi and Connors. ${ }^{17}$ Excess quantities of the drug were transferred to batches of $25 \mathrm{ml}$ of aqueous solution of $\beta-\mathrm{CD}$ in various molar concentrations $(2.0,4.0,6.0,8.0,10.0,12.0$ and 14.0 $\mathrm{mmol} / \mathrm{L}$ ) contained in screw capped vials. These solutions were stirred on electromagnetic stirrer (Remi) at a constant temperature $37^{\circ} \mathrm{C} \pm 0.1$ for $48 \mathrm{~h}$ and $400 \mathrm{rpm}$ (this duration was previously tested to be sufficient to reach equilibrium). The samples were filtered through a $0.22 \mu \mathrm{m}$ membrane filter. The filtrate was suitably diluted and analyzed spectrophotometrically for drug content at the wavelength of $238.2 \mathrm{~nm}$ using a U.V. visible spectrophotometer, Shimazdu-1601. Solubility studies were performed in triplicate. Data obtained from phase solubility diagram was used to determine stoichiometric ratio by plotting concentration of LOV against the concentration of $\beta-\mathrm{CD}$. The stability constant (Ks) for the complex was determined from the graph using equation. ${ }^{17}$

$\mathrm{Ks}=\frac{\text { slope }}{\mathrm{S}_{\mathrm{o}}(1-\text { slope })}$

where slope is obtained from the graph and $\mathrm{S}_{0}$ is the equilibrium solubility of Lov in water.

Preparation of inclusion complexes. Complex of $\beta-C D$ and Lov were prepared in the molar ratio of 1:1 (on the basis of phase solubility study) by different methods like kneading, coevaporation, and physical mixture. For preparation of the complex by coevaporation method (CCOE), methanol and water were used as solvents. The required quantity of Lov and $\beta-C D$ were dissolved in methanol and water respectively. Both the solutions were mixed and solvents were evaporated by controlled heating at 45 $-50{ }^{\circ} \mathrm{C}$. The resultant solid was pulverized and then sieved through 120 \#. Complex prepared by kneading method (CKD) was prepared by geometric mixing of powders, Lov and $\beta-C D$, and then kneaded with $1: 1$ mixture of ethanol - water to obtain a mass with a 
pasty consistency, which was dried in a hot air oven at 45 to $50{ }^{\circ} \mathrm{C}$. The dried mass was sieved through 120 \#. Physical mixture (PM) of $\beta$-CD and Lov was prepared by geometric mixing of Lov and $\beta-\mathrm{CD}$ without applying pressure. The required quantities of Lov and $\beta-C D$ were taken in a glass mortar and mixed for 15 minutes.

\section{Characterization of Complexes}

Fourier transform infrared (FTIR) spectroscopic analysis. The Fourier transform infrared spectrum of moisture free powdered sample of Lov, $\beta-\mathrm{CD}, \mathrm{PM}, \mathrm{CCOE}$, and CKD were recorded on IR spectrophotometer (FTIR - 8300, Shimadzu) by potassium bromide $(\mathrm{KBr})$ pellet method.

X-ray diffraction (XRD) analysis. Powder diffraction patterns of all samples were obtained using Phillips PW 3710 scanner, IW 1830 generator with a $\mathrm{CuK} \alpha$ anode at $40 \mathrm{kV}$ and $30 \mathrm{~mA}$ and at a scan rate of $1^{\circ} \mathrm{min}^{-1}$ with $2 \theta$ range from 1 to $40^{\circ}$, conducted at Sun Pharmaceutical Advance Research Center, Vadodara, India.

Differential scanning calorimetry (DSC) analysis. DSC scans of the powdered sample of all samples were recorded using DSC- Shimadzu 60 with a TDA trend line software. The thermal traces were obtained by heating the complex from 50 to $300{ }^{\circ} \mathrm{C}$ at heating rate of $10{ }^{\circ} \mathrm{C}$ under inert nitogen dynamic atmosphere $(100 \mathrm{ml} / \mathrm{min})$ in open aluminum crucibles and were conducted at Pharmacy Department, M. S. University, Baroda, India.

Dissolution Studies. Dissolution studies of Lov in powder form, PM, CCOE, and CKD were performed to evaluate in vitro drug release profile. Dissolution studies were carried out using a USP dissolution apparatus type II with $500 \mathrm{ml}$ dissolution mediums at $37{ }^{\circ} \mathrm{C} \pm 0.5$ and $50 \mathrm{rpm}$ for $3 \mathrm{~h} .0 .1 \mathrm{~N}$ $\mathrm{HCl}$ and Phosphate buffer (pH 6.8) containing $0.25 \%$ $(\mathrm{w} / \mathrm{v})$ of sodium lauryl sulfate (SLS) were used as different dissolution mediums. At fixed time intervals (every $15 \mathrm{~min}$ for $3 \mathrm{~h}$.), $10 \mathrm{ml}$ aliquots were withdrawn, filtered, suitably diluted and then assayed for Lov content by measuring the absorbance at 238.2 $\mathrm{nm}$. Fresh media $(10 \mathrm{ml})$, which was prewarmed at
$37{ }^{\circ} \mathrm{C}$, was replaced in to the dissolution medium after each sampling to maintain its constant volume throughout the test. Pure drug, PM, CCOE, and CKD were evaluated for dissolution rate studies. Dissolution studies were performed in five replicates $(\mathrm{n}=5)$, and calculated mean values of cumulative drug release were used while plotting the release curves. MDT values were calculated to compare the extent of improvement in the dissolution rate of PM, CCOE, and CKD.

Formulation studies. Tablets containing $10 \mathrm{mg}$ of Lov were made by direct compression using compressible lactose $(60 \% \mathrm{w} / \mathrm{w})$, maize starch $(23 \%$ $\mathrm{w} / \mathrm{w})$, sodium starch glycollate $(5 \% \mathrm{w} / \mathrm{w})$, colloidal silicon dioxide $(1 \% \mathrm{w} / \mathrm{w})$, and magnesium stearate $(1 \% \mathrm{w} / \mathrm{w})$, as excipients. Tablets containing CKD equivalent to $10 \mathrm{mg}$ Lov were made similarly but excluding lactose. The tablets were studied in five replicates for release profile of drug using the same methodology as described in in vitro dissolution studies.

Statistical analysis. The similarity factor $f_{2}$ is a measure of similarity in the percentage dissolution between two dissolution curves and is defined by following equation (12):

$$
f_{2}=50 \log \left\{\left[1+\frac{1}{n} \sum_{t=1}^{n} w_{t}\left(R_{t}-T_{t}\right)^{2}\right]^{-0.5} x 100\right\}
$$

where $\mathrm{n}$ is the number of withdrawal points, $R_{t}$ is the percentage dissolved of the reference at the time point $t$ and $T_{t}$ is the percentage dissolved of the test at the time point $t$.

A value of $100 \%$ for the similarity factor $\left(f_{2}\right)$ suggests that the test and reference profiles are identical. Values between 50 and 100 indicate that the dissolution profiles are similar whilst smaller values imply an increase in dissimilarity between release profiles. ${ }^{12}$

\section{RESULTS AND DISCUSSION}

Phase solubility study. The solubility of Lov in water at $25{ }^{\circ} \mathrm{C}$ is $0.4 \mu \mathrm{g} / \mathrm{ml}$; therefore, Lov can be considered to be a water insoluble drugs. The phase 
solubility curve of Lov in the presence of $\beta-C D$ is shown in Figure 1. From this curve, it can be seen that the apparent solubility of Lov increases due to the formation of an inclusion complex between Lov and $\beta-\mathrm{CD}$. A linear increase of solubility of Lov was observed with an increase in concentration of $\beta-\mathrm{CD}$ in water. Solubility of Lov is increased by 10.75 -fold at $14 \mathrm{mmol} / \mathrm{L}$ concentration of $\beta-\mathrm{CD}$. An indication of the process of transfer of Lov from pure water to aqueous solution of $\beta-\mathrm{CD}$ was obtained from the values of Gibbs free energy change. The Gibbs free energy of transfer $\left(\Delta \mathrm{G}_{\mathrm{tr}}{ }^{\circ}\right)$ of Lov from pure water to aqueous solution of $\beta-\mathrm{CD}$ was calculated using ${ }^{18}$ equation

$$
\Delta \mathrm{G}_{\mathrm{tr}}{ }^{\circ}=-2.303 R T \log \left(\frac{\mathrm{S}_{\mathrm{o}}}{\mathrm{S}_{\mathrm{s}}}\right)
$$

where So/Ss $=$ the ratio of molar solubility of Lov in aqueous solution of $\beta-C D$ to that of the pure water. The obtained values of Gibbs free energy are shown in Table 1. $\Delta \mathrm{G}_{\mathrm{tr}}{ }^{\circ}$ values were all negative for $\beta-\mathrm{CD}$ at various concentrations, indicating the spontaneous nature of Lov solubilization, and it decreased with an

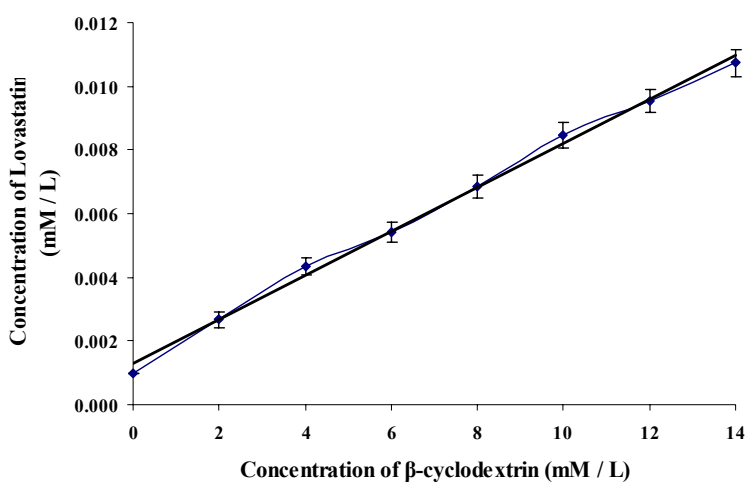

Figure 1. Phase Solubility Curve of Lov in Aqueous Solution of $\beta$ $\mathrm{CD}$ at $37^{\circ} \mathrm{C}$.

Table 1. Gibbs free energy of transfer $\left(\Delta G \operatorname{tr}^{\circ}\right)$ for solubilization process of Lov in aqueous solutions of $\beta$-CD at $37^{\circ} \mathrm{C}$.

\begin{tabular}{cc}
\hline$\%(\mathrm{w} / \mathrm{v})$ of $\beta$-CD in water & $\Delta \mathrm{G}_{\mathrm{tr}}{ }^{\circ}(\mathrm{KJ} / \mathrm{mol})^{\mathrm{a}}$ \\
\hline 0.23 & -2.56 \\
0.45 & -3.81 \\
0.68 & -4.39 \\
0.91 & -4.99 \\
1.14 & -5.53 \\
1.36 & -5.85 \\
1.59 & -6.15 \\
\hline
\end{tabular}

increase in its concentration, demonstrating that the reaction became more favorable as the concentration of $\beta$-CD increased.

The phase solubility plot showed an $\mathrm{A}_{\mathrm{L}}$ type solubility curve, which indicates that only $1: 1 \beta-\mathrm{CD}$ Lov inclusion complex was formed in solution. The stability constant (Ks) calculated for the complexation of Lov with $\beta$-CD in solution at $25{ }^{\circ} \mathrm{C}$ is $538.84 \mathrm{M}^{-1}$, assuming a $1: 1$ stoichiometry for the complex, which indicates a suitable and stable complex formation. Ks value between 200-5000 $\mathrm{M}^{-1}$ is considered as the most suitable for the improvement of bioavailability of poorly soluble drugs. ${ }^{17}$

\section{Characterization of complexes}

Fourier transform infrared (FTIR) spectroscopic analysis. The FT-IR spectra of PM, CCOE and CKD were compared with spectrum of $\beta-C D$ and Lov (Figure 2). The presence or absence of characteristic peaks associated with specific structural groups of the drug molecule were noted. The spectrum of pure Lov presented characteristic peaks at $3510 \mathrm{~cm}^{-1}$ (Alcohol O-H stretching vibration), $3016 \mathrm{~cm}^{-1}$ (Olefinic C-H stretching vibration), 2970, 2930 and $2876 \mathrm{~cm}^{-1}$ (Methyl and Methylene C-H stretching vibration), 1725, 1713 and $1690 \mathrm{~cm}^{-1}$ (Lactone and ester carbonyl strech (Hydrogen bonded for 1711 and $1700 \mathrm{~cm}^{-1}$ )), 1430, 1378 and $1350 \mathrm{~cm}^{-1}$ (Methyl and Methylene bending vibration), 1275, 1228, 1080 and $1050 \mathrm{~cm}^{-1}$ (Lactone and Ester C-O-C bending vibration), $972 \mathrm{~cm}^{-1}$ (Alcohol C-OH strech), and $873 \mathrm{~cm}^{-1}$ (Trisubstituted olefinic C-H wag), respectively. The spectra of PM, CCOE and CKD were equivalent to the addition spectrum of $\beta-C D$ and Lov. These results indicate absence of well defined chemical interaction between $\beta-\mathrm{CD}$ and Lov during mixing, coevaporation and kneading. IR spectra of PM, CCOE and CKD showed most of characteristic peaks similar to that of $\beta$-CD except one peak at $1700 \mathrm{~cm}^{-1}$ for lactone and ester carbonyl stretching vibration (Hydrogen bonded for 1711 and $1700 \mathrm{~cm}^{-1}$ ) which is characteristic of Lov. This indicates that pyrol part of the Lov remains 
outside the $\beta-C D$ whereas the remaining part fits inside the $\beta$-CD cavity.

Differential scanning calorimetry (DSC) analysis. The thermograms for pure Lov, $\beta-\mathrm{CD}, \mathrm{PM}$, CCOE, and CKD are presented in Figure 3. The Lov showed a melting endotherm at $173.7{ }^{\circ} \mathrm{C}$ with enthalpy of fusion $(\Delta \mathrm{H}) \quad 104.282 \mathrm{~J} / \mathrm{g}$. In the thermogram of the $\beta-\mathrm{CD}$, two endothermic peaks were present. Peak at $100^{\circ} \mathrm{C}$ was due to loss of water from $\beta-\mathrm{CD}$ molecules and peak at $300^{\circ} \mathrm{C}$ indicated the melting or thermal decomposition of $\beta$-CD. A characteristic sharp endothermic peak of Lov in the range of 171 to $176{ }^{\circ} \mathrm{C}$ was absent in the thermograms of CCOE and $\mathrm{CKD}$, indicating partial amorphization of the drug and trapping of Lov inside the $\beta$-CD cavity. In the thermogram of PM, sharp endotherm was observed at the same position to that of Lov indicating presence of untrapped Lov.

X-ray diffraction (XRD) analysis. The diffraction spectrums of pure Lov, PM, CCOE and CKD are presented in Figure 4. The diffraction spectrum of pure Lov showed that the drug was of crystalline nature as indicated by numerous, distinct peaks. Numerous prominent diffraction peaks of Lov were observed indicating the presence of crystalline Lov. Pure $\beta$-CD showed characteristic peaks at $2 \theta$ of $7.92,9.41,10.88,15.7,16.69,18.9,26.06,28.38$, etc. The CCOE and CKD exhibited the presence of characteristic peaks of $\beta-C D$ and absence of characteristic peaks of Lov, suggesting that Lov is trapped inside the cavity of $\beta$-CD. However, peaks at $2 \theta$ between 20.00 to 25.00 in complexes corresponding to Lov indicate partial untrapped part of Lov inside the $\beta-C D$ cavity which also conforms with Infrared study of complexes. The spectrums of CCOE and CKD were characterized by absence of prominent peaks of Lov, suggesting that Lov is present in amorphous form. Moreover, no other peaks than those that could be assigned to the pure $\beta-C D$ and Lov were detected in the complexes, indicating absence of chemical interaction in the solid state between the two entities. Results of this study confirms that Lov is no longer present as a crystalline material and its $\beta-\mathrm{CD}$ complexes exist in amorphous state.

Dissolution studies. The dissolution of poorly soluble drugs requires dissolution media that are different from those normally used for water soluble drugs. One of the techniques that has been used is the incorporation of a small amount of surfactant in the dissolution medium. ${ }^{19}$ Studies on sodium lauryl sulfate (SLS) solutions indicate that surface tension of SLS solutions decreased dramatically above the critical micelle concentration $(0.023 \%)$, and it reached a minimum surface tension at $0.2 \%$ with no significant change at higher concentrations. ${ }^{20,21}$ This suggested that a biocomparable surface activity can be achieved at low surfactant concentrations $(0.2 \%)$. Since the principal objective of this work was to improve the dissolution rate of Lov, dissolution studies were carried out for initially $3 \mathrm{~h}$.

$\mathrm{Q}_{30 \text { min }}, \mathrm{Q}_{60 \text { min }}$ and $\mathrm{Q}_{120 \text { min }}$ values (percent drug dissolved within 120 minutes) in $0.1 \mathrm{~N} \mathrm{HCl}$ and phosphate buffer ( $\mathrm{pH}$ 6.8) are reported in Table 2. From this data, it is evident that onset of dissolution of pure Lov is very low in both dissolution medium (29.99 and $27.20 \%$ within 120 min, respectively). CKD and CCOE considerably enhanced dissolution rates within 120 min compared to pure Lov and PM. The graphical presentation of the dissolution profile of pure Lov, PM, CCOE and CKD samples in $0.1 \mathrm{~N}$ $\mathrm{HCl}$ and phosphate buffer ( $\mathrm{pH}$ 6.8) over a period of 3 $\mathrm{h}$ is shown in Figures 5 and 6, respectively. CKD and CCOE enhanced dissolution rate of Lov significantly (90-100\% in $0.1 \mathrm{~N} \mathrm{HCl}$ and $85-95 \%$ in phosphate buffer) within $3 \mathrm{~h}$. Possible mechanism of improved dissolution rates of complexes have been proposed by Vromans et al. ${ }^{22}$ and include: reduction of crystallite size, a solubilization effect of carrier, absence of aggregation of drug crystallites, improved wettability, dispersibility of a drug from dispersion, dissolution of the in the hydrophilic carrier, conversion of drug to amorphous state, and finally, the combination of the above methods. ${ }^{22}$

The dissolution rate of Lov from PM was higher (74.71 \% in $0.1 \mathrm{~N} \mathrm{HCl}$ and $74.72 \%$ in phosphate buffer) than that of pure Lov $(36.10 \%$ in $0.1 \mathrm{~N} \mathrm{HCl}$ 

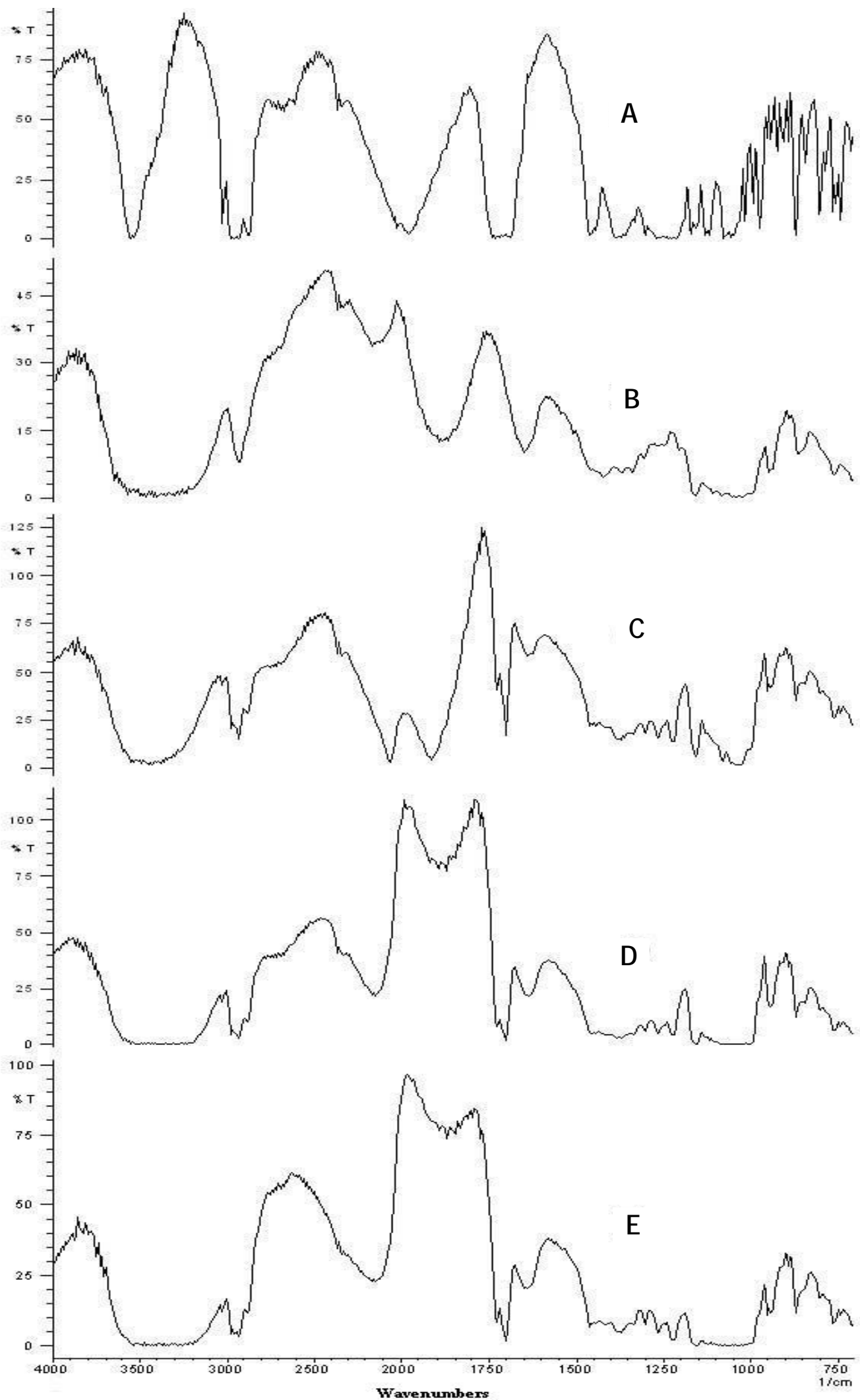

Figure 2. FTIR Spectrograms of Lov (A), $\beta$-CD (B), PM (C), CCOE (D) and CKD (E). 


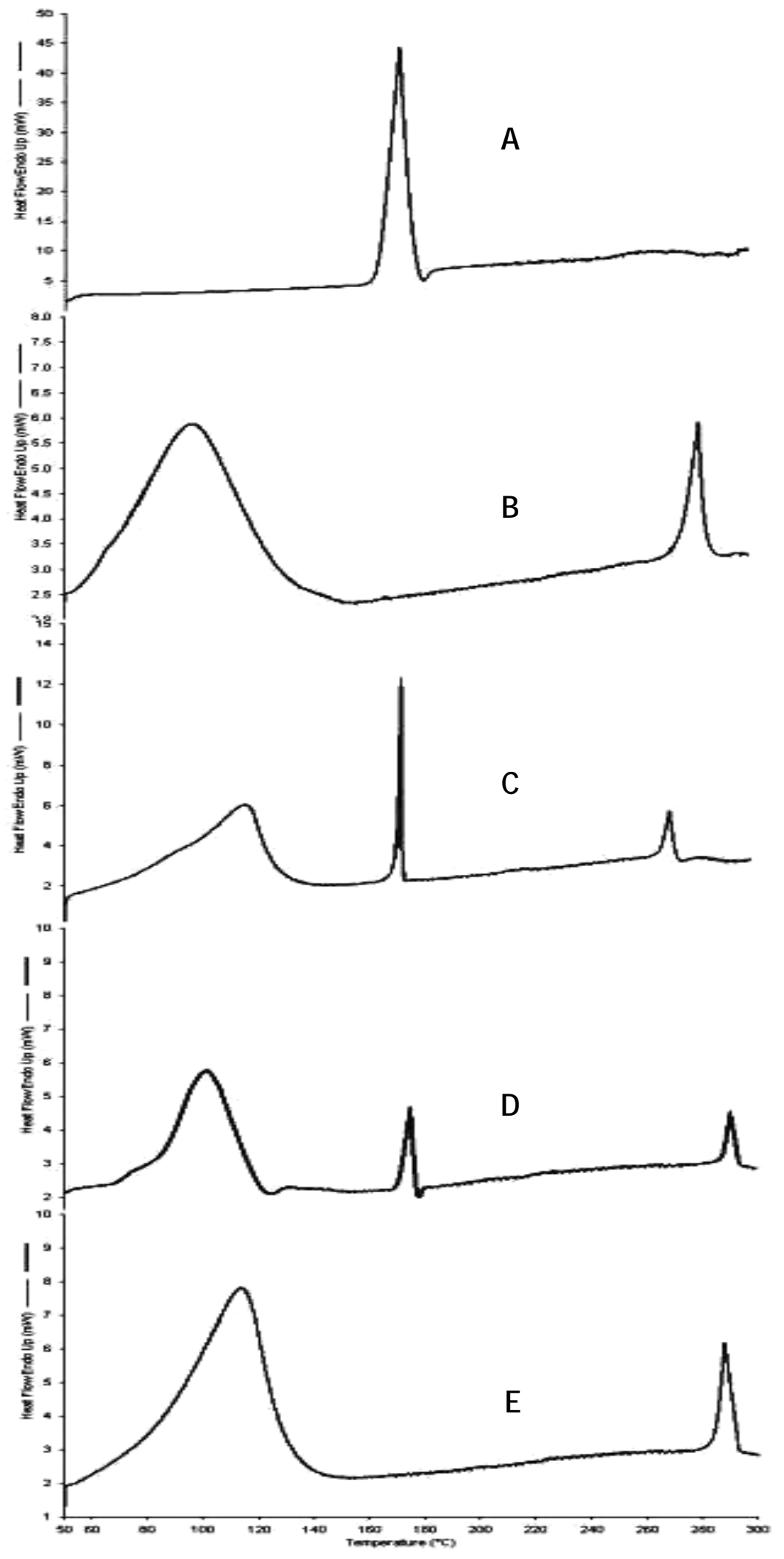

Figure 3. DSC thermograms Lov (A), $\beta-\mathrm{CD}(\mathrm{B}), \mathrm{PM}(\mathrm{C}), \mathrm{CCOE}$ (D) and CKD (E). 


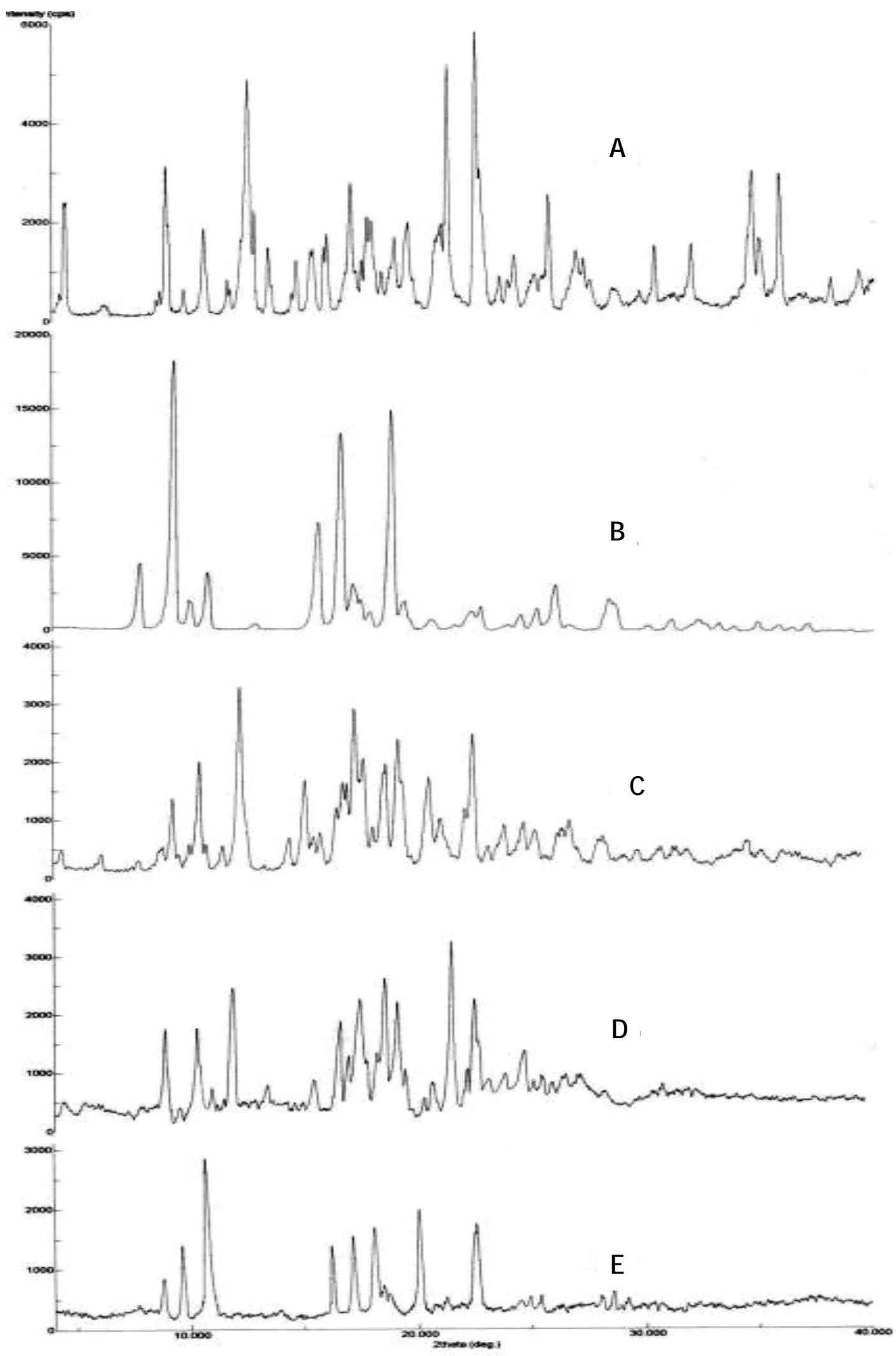

Figure 4. X-Ray Diffractograms of Lov (A), $\beta$-CD (B), PM (C), CCOE (D) and CKD (E) 
and $34.00 \%$ in phosphate buffer) within $3 \mathrm{~h}$. The increased dissolution rate observed in case of PM can be attributed to several factors such as a solubilization effect of $\beta-C D$, improved wettability of drug, and prevention of particle aggregation.

MDT reflects the time for the drug to dissolve and is the first statistical moment for the cumulative dissolution process that provides an accurate drug release rate. ${ }^{13}$ It is accurate expression for drug release rate. A higher MDT value indicates greater drug retarding ability. In order to calculate mean dissolution time (MDT) of pure Lov, PM, CCOE, and $\mathrm{CKD}$, the mean $(\mathrm{n}=3)$ of cumulative drug release $(\mu \mathrm{g})$ was used. The obtained values of MDT for pure Lov, PM, CCOE, and CKD are presented in Table 3. The MDTs of Lov are $64.92 \mathrm{~min}$ in $0.1 \mathrm{~N} \mathrm{HCl}$ and $66.13 \mathrm{~min}$ in phosphate buffer. These values decreased greater extent to after preparing complex of Lov with $\beta$-CD i.e. $26.20 \mathrm{~min}$ in $0.1 \mathrm{~N} \mathrm{HCl}$ and $32.67 \mathrm{~min}$ in phosphate buffer for CKD and 38.42 $\min$ in $0.1 \mathrm{~N} \mathrm{HCl}$ and $44.29 \mathrm{~min}$ in phosphate buffer for $\mathrm{CCOE}$.

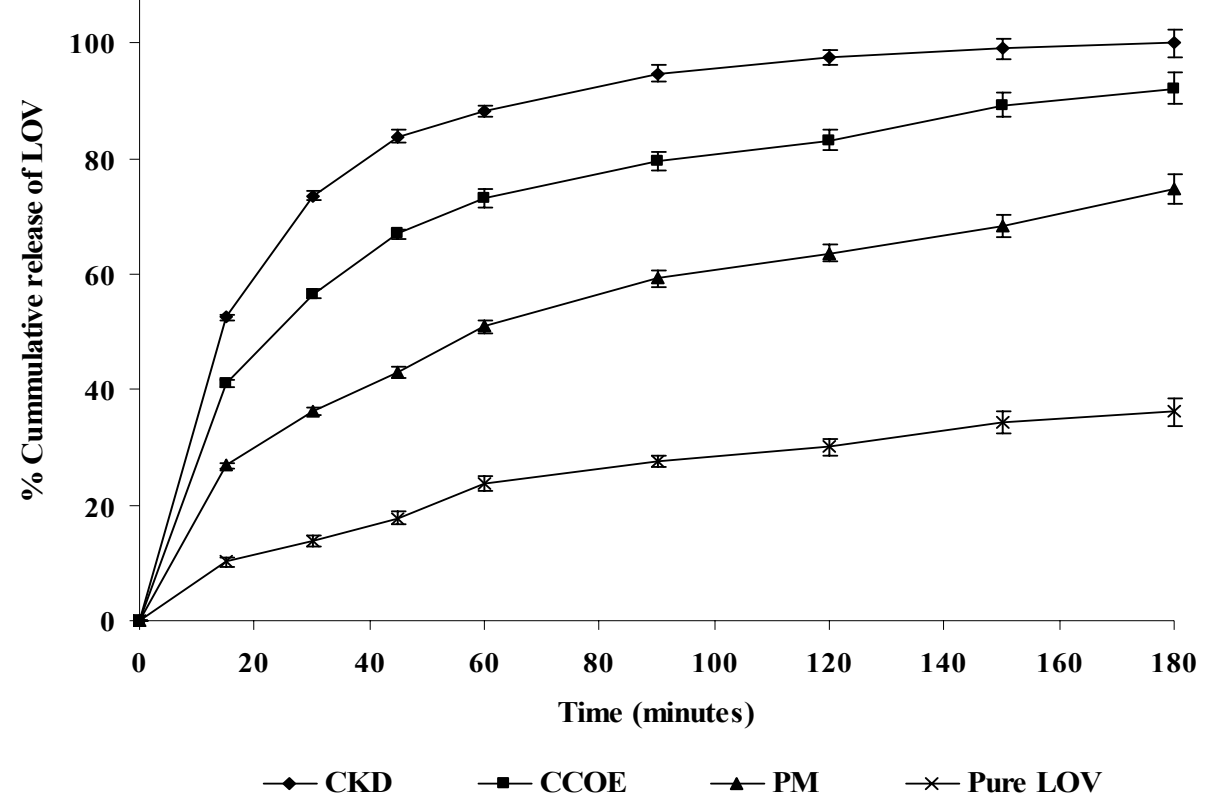

Figure 5. In vitro Dissolution Profiles of pure Lov, and its Physical Mixture and Complexes in $0.1 \mathrm{~N} \mathrm{HCl}$ (Each point is Mean $\pm \mathrm{SD}$ of five experiments)

Table 2. Percentage Drug Dissolved Within 120 Minutes

\begin{tabular}{lcccccc} 
& \multicolumn{5}{c}{ \% Lov release $^{\mathrm{a}}$} \\
\cline { 2 - 7 } Sample & \multicolumn{3}{c}{$0.1 \mathrm{~N} \mathrm{HCl}$} & \multicolumn{3}{c}{ Phosphate buffer $(\mathrm{pH} 6.8)$} \\
\cline { 2 - 7 } & $\mathrm{Q}_{30 \min }$ & $\mathrm{Q}_{60 \min }$ & $\mathrm{Q}_{120 \min }$ & $\mathrm{Q}_{30 \min }$ & $\mathrm{Q}_{60 \min }$ & $\mathrm{Q}_{120 \min }$ \\
\hline Pure Lov & $13.75 \pm 0.93$ & $23.63 \pm 1.33$ & $29.99 \pm 1.42$ & $10.62 \pm 0.89$ & $18.60 \pm 1.42$ & $27.20 \pm 1.59$ \\
PM & $36.35 \pm 0.67$ & $50.87 \pm 1.05$ & $63.59 \pm 1.38$ & $30.9 \pm 0.96$ & $44.09 \pm 1.00$ & $62.77 \pm 1.76$ \\
CCOE & $56.55 \pm 0.78$ & $73.12 \pm 1.68$ & $83.11 \pm 1.77$ & $46.29 \pm 0.47$ & $68.04 \pm 1.53$ & $79.69 \pm 1.74$ \\
CKD & $73.58 \pm 0.88$ & $88.33 \pm 0.96$ & $97.41 \pm 1.23$ & $60.79 \pm 0.93$ & $81.45 \pm 1.27$ & $92.44 \pm 1.34$ \\
\hline
\end{tabular}

${ }^{\mathrm{a}} \mathrm{Mean} \pm \mathrm{SD}, \mathrm{n}=5$.

Calculated $f_{2}$ values for all samples are presented in Table 4. From this Table, it is evident that the release profile of Lov from CKD is highly different from pure Lov in both dissolution medium $\left(f_{2}\right.$ values 10.97 in $0.1 \mathrm{~N} \mathrm{HCl}$ and 13.17 in phosphate buffer).
Even release profiles of Lov from CCOE and PM are also significantly different from pure Lov in both dissolution mediums. The release profile of Lov from CCOE and PM were similar in both the dissolution 
medium as the $f_{2}$ values for these profiles are greater to 50 .

Table 3. Mean Dissolution time (MDT) values for pure Lov, PM, CCOE, and CKD in $0.1 \mathrm{~N} \mathrm{HCl}$ and Phosphate Buffer (pH 6.8)

\begin{tabular}{lcc}
\hline & \multicolumn{2}{c}{ MDT (min) ${ }^{\mathrm{a}}$} \\
\cline { 2 - 3 } Sample & $0.1 \mathrm{~N} \mathrm{HCl}$ & Phosphate Buffer (pH 6.8) \\
\hline Pure Lov & 64.92 & 66.13 \\
PM & 51.60 & 58.49 \\
CCOE & 38.42 & 44.29 \\
CKD & 26.20 & 32.67 \\
\hline
\end{tabular}

${ }^{\mathrm{a}}$ Mean $\pm \mathrm{SD}, \mathrm{n}=5$.
Table 4. Similarity Factor $\left(f_{2}\right)$ for Release Profiles of Lov in 0.1 N HCl and Phosphate (pH 6.8).

Release profile in $0.1 \mathrm{~N} \mathrm{HCL}$

\begin{tabular}{llcccc} 
Release & Sample & Lov & PM & CCOE & CKD \\
\cline { 2 - 6 } & Lov & 81.41 & 27.55 & 17.87 & 13.17 \\
& PM & 26.71 & 66.8 & 39.34 & 28.21 \\
& CCOE & 16.03 & 36.14 & 53.04 & 46.99 \\
& CKD & 10.97 & 24.88 & 44.22 & 48.82 \\
\hline
\end{tabular}

Formulation studies. The complex prepared by kneading method was studied for physical properties to judge its tableting ability. In general, compressibility index values up to $15 \%$ and angle of repose between 25 to $30^{\circ}$ results in good to

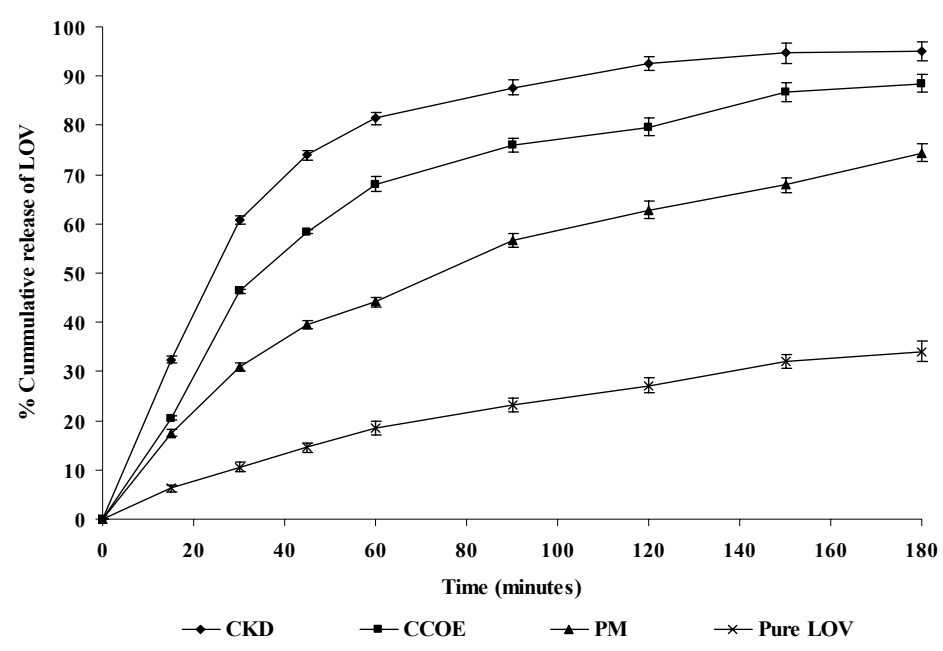

Figure 6. In vitro Dissolution Profiles of pure Lov, and its Physical Mixture andComplexes in Phosphate Buffer (pH 6.8) (Each point is Mean \pm SD of five experiments)

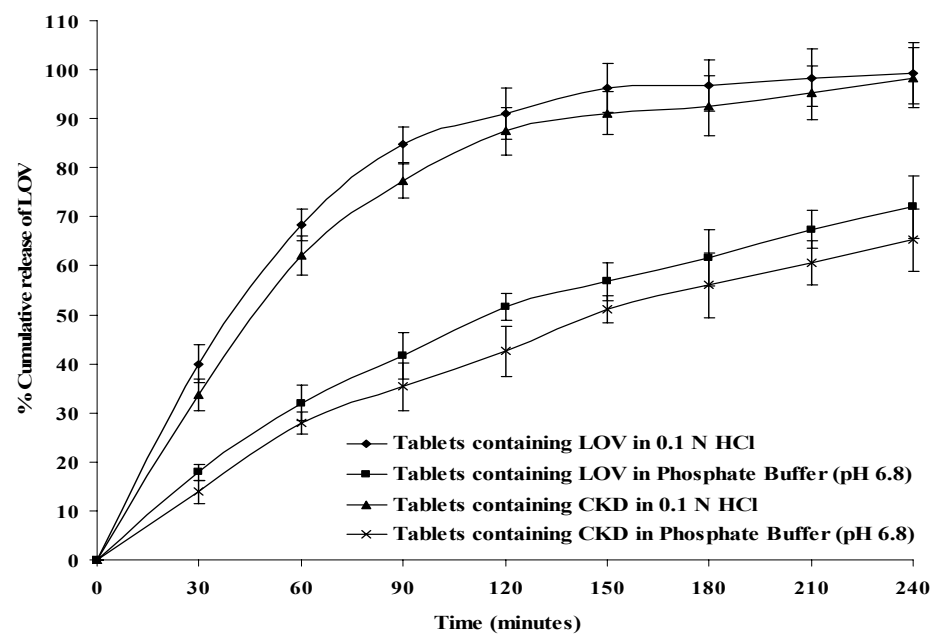

Figure 7. Comparative Drug Release Profiles of Conventional Tablets Containing Lov and Tablets Containing CKD in $0.1 \mathrm{~N} \mathrm{HCl}$ and Phosphate Buffer $\mathrm{pH}$ (6.8) (Each point is Mean \pm SD of five experiments). 
excellent flow properties. ${ }^{23}$ Percentage of compressibility and angle of repose of CKD were $10.23 \%$ and $28.9^{\circ}$, respectively. These values indicate good compressibility and flow properties, making it suitable for tableting.

Tablets prepared using complex showed faster and reproducible release as compared to the tablets containing pure Lov and no $\beta-\mathrm{CD}$, which showed 72 and $65.3 \%$ release in 4 hours with $\mathrm{T}_{50 \%}$ of 115 minutes and $145 \mathrm{~min}$ in $0.1 \mathrm{~N} \mathrm{HCl}$ and phosphate buffer, respectively (Figure 7). This confirmed the advantage of improved aqueous solubility of Lov in its complex form, which can be formulated as tablets with better dissolution characteristic. Release profiles of Lov from conventional tablets containing Lov are significantly different from tablets containing CKD as the $f 2$ values are 26.14 and 25.56 in $0.1 \mathrm{~N} \mathrm{HCl}$ and phosphate buffer, respectively. MDT values of Lov from tablets containing CKD in both dissolution medium $(51.07 \mathrm{~min}$ in $0.1 \mathrm{~N} \mathrm{HCl}$ and $60.49 \mathrm{~min}$ in phosphate buffer) are significantly lower than that of conventional tablets containing only Lov and no $\beta$ $\mathrm{CD}(87.96 \mathrm{~min}$ in $0.1 \mathrm{~N} \mathrm{HCl}$ and $92.96 \mathrm{~min}$ in phosphate buffer).

\section{CONCLUSION}

An inclusion complex of Lov and $\beta-C D$ was prepared successfully by kneading and coevaporation method in a molar ratio of $1: 1$. This was confirmed by FTIR, DSC, and X-ray diffraction spectroscopy. The complexes prepared by different methods help to improve aqueous solubility and in-vitro release profile. These findings are extremely important from a commercial point of view as the prepared complex removes draw back of poor dissolution profile of Lov.

\section{ACKNOWLEDGEMENT}

We would like to thank Lincoln Pharmaceuticals Ltd. for donating Lovastatin. We would also like to thank Maan Pharmaceuticals Ltd. for providing formulation excipients. We are grateful to the Department of Pharmacy, M. S. University, India for conducting DSC studies of the samples. We are thankful to Sun Pharmaceutical Advance Research Center, India for conducting XRD studies of the samples.

\section{REFERENCES}

1. Pitha, J., Milecki, J., Fales, H., Pannell, L. and Uekama, K. 1986. Hydroxypropyl- $\beta$-cyclodextrin: Preparation and characterization; Effects on solubility of drugs. Int. J. Pharm. 29, 73-82.

2. Duchêne, D. 1987. SS. Cyclodextrins and Their Industrial Uses Editions de Santé Paris, pp. 447-460.

3. Baboota, S., and Agarwal, S.P. 2003. Rofecoxib complexation with $\beta$-cyclodextrin: Influence on the antiinflammatory and ulcerogenic activity. Die Pharmazie 58, 73-74.

4. Fernandes, C.M., Teresa, V. M. and Veiga, F.J. 2002. Physicochemical characterization and in vitro dissolution behavior of nicardipine-cyclodextrins inclusion compounds. Eur. J. Pharm. Sci. 15, 79-88.

5. Kamada, M., Hirayama, F., Udo, K., Yano, H., Arima, H., and Uekama, K. 2002. Cyclodextrin conjugate-based controlled release system: repeated- and prolonged-releases of ketoprofen after oral administration in rats. J. Control. Release. 82, 407-416.

6. Mukne, A.P. and Nagarsenker, M.S. 2004. Triamterene- $\beta$ cyclodextrin systems: Preparation, characterization and in vivo evaluation. AAPS PharmSci Tech. 5, article 19.

7. Uekama, K., and Otagiri, M. 1987. Cyclodextrins in drug carrier systems. Crit. Rev. Ther. Drug Carrier Syst. 3, 1-40.

8. Ghorab, M.K., and Adeyeye, M.C. 2001 Elucidation of solution state complexation in wet-granulated oven-dried ibuprofen and $\beta$-cyclodextrin: FT-IR and 1H-NMR studies. Pharm. Dev. Technol. 6, 315-324.

9. Veiga, F.J., Fernandes, C., Carvalho, R.A. and Geraldes, C.F. 2001. Molecular modeling and 1H-NMR: ultimate tools for the investigation of tolbutamide: $\beta$-cyclodextrin and tolbutamide: hydroxypropyl- $\beta$-cyclodextrin complexes. Chem. Pharm. Bull (Tokyo). 49, 1251-1256.

10. Bekers, O., Beijnen, J.H., Otagiri, M., Bult, A. and Underberg, W.J. 1990. Inclusion complexation of doxorubicin and daunorubicin with cyclodextrins. J. Pharm. Biomed. Anal. 8, 671-674.

11. Uekama, K., Fujinaga, T., Hirayama, F., Otagiri, M., Yamasaki, M., Seo, H., Hashimoto, T. and Tsuruoka, M. 1983. Improvement of the oral bioavailability of digitalis glycosides by cyclodextrin complexation. J. Pharm. Sci. 72, 1338-1341.

12. Moore, J.W. and Flanner, H. 1996. Mathematical comparison of dissolution profiles. Pharm. Tech. 20, 64-74. 
13. Reppas, C. and Nicolaides, E. 2000. Analysis of drug dissolution data, In: Oral Drug Absorption Prediction and Assessment (Dressman, J.B., and Lennernäs, H., Eds.) Marcel Dekker, Inc.: New York, pp. 229-254.

14. MacDonald, J.S., Gerson, R.J., Kornburst, D.J., Kloss, M.W., Prahalada, S., Berry, P.H., Alberts, A.W., and Bokelman, D.L. 1998. Preclinical evaluation of lovastatin. Am. J. Cardiol. 62, 16J-27J.

15. Slater, E.E., and MacDonald, J.S. 1998. Mechanism of action and biological profile of HMG CoA reductase inhibitors: a new therapeutic alternative. Drugs. 36 (Suppl. 3), 72-82.

16. Gerald, S.B., Dean, K.E. and Micheal J.K. 1992. Lovastatin. In: Analytical Profiles of Drug Substances and Excipients, Harry G. B. vol 21, Academic press, Inc., New York, pp. 277-315.

17. Higuchi, T. and Connors, K. 1965. Phase solubility techniques. Advances in Analytical Chemistry and Instrumention, 4, 17-123.

18. Chengsheng, L., Chenguang, L., and Kashappa Goud H. D. 2005. Enhancement of dissolution rate of valdecoxib using solid dispersions with polyethylene glycol 4000. Drug Development \& Industrial Pharmacy. 1, 1-10.
19. Serajuddin, A.T. M., Sheen, P. C. and Augustine, M.A. 1990. Improved dissolution of poorly water soluble drug from solid dispersions in polyethylene: polysorbate 80 mixture. $J$. Pharamceutical Sci. 79, 463-464.

20. Barzegar-Jalali, M., Maleki, N., Garjani, A., Khandar, A. A., Haji-Hosseinloo, M., Jabbari, R.C. and Dasmalchi, S. 2002. Enhancement of dissolution rate and anti-inflammatory effects of piroxicam using solvent deposition technique. Drug Development \& Industrial Pharmacy. 28, 681-686.

21. Tang, L., Khan, S.U. and Muhmmad, N.A. 2001. Evaluation and selection of bio-relevant dissolution media for a poorly water soluble new chemical entity. Pharmaceutical Development Technology. 6, 531-540.

22. Vromans, H., Eisson, A. C. and Coenraad, F.L.. 1989. Mechanism of dissolution of drug-cyclodextrin complexes. Drug Dev. Ind. Pharm. 15, 250-255.

23. Aulton, M.E. 1988. Pharmaceutical Technology. In: Pharmaceutics: The Science of Dosage Form Design. London, UK: Churchill Livingstone; pp. 600-616. 